Hautarzt 2010 • 61:1012-1013

DOI 10.1007/s00105-010-2014-8

Online publiziert: 20. November 2010

(c) Springer-Verlag 2010

\author{
M. Sticherling ${ }^{1} \cdot$ D. Bruch-Gerharz ${ }^{2}$ \\ ${ }^{1}$ Hautklinik, Universitätsklinikum Erlangen \\ ${ }^{2}$ Hautklinik, Universitätsklinikum Düsseldorf
}

\title{
Haut und Schwangerschaft
}

Die Schwangerschaft gehört zu den natürlichsten Zuständen einer Frau, die nur selten zu schweren Erkrankungen führt oder von diesen begleitet wird. Vielmehr ist der weibliche Körper in der Lage, sehr effizient eine natürliche Transplantationssituation ohne Schaden für die Mutter oder das werdende Kind zu bewältigen. Die vielfältigen Veränderungen während der Schwangerschaft ziehen auch das Hautorgan mit ein, sodass sich eine Reihe physiologischer, gelegentlich aber auch krankhafter Hautveränderungen manifestieren kann. Die isoliert in der Schwangerschaft auftretenden Dermatosen sind insgesamt selten und bereiten manchmal differenzialdiagnostische Schwierigkeiten, sie von anderen, nicht-schwangerschaftstypischen Hauterkrankungen abzugrenzen. Ihre Diagnostik und Therapie ist aus ethischen und medizinischen Gründen eingeschränkt, andererseits können sie zu einer massiven Beeinträchtigung der Lebensqualität der Schwangeren, aber auch zur Gefährdung des werdenden Kindes führen und bedürfen daher einer zügigen und korrekten Einordnung. Die genauen Pathomechanismen dieser Erkrankungen sind bis heute nicht geklärt, was auch eine gezielte Therapie einschränkt. Frau Priv.-Doz. Dr. AmbrosRudolph beschreibt in ihrem Beitrag zu diesem Schwerpunktheft die klinischen Bilder sowie therapeutischen Möglichkeiten der sog. Schwangerschaftsdermatosen und arbeitet dabei wichtige differenzialdiagnostische Charakteristika der einzelnen Erkrankungen heraus.

Neben genuinen, nur in der Schwangerschaft auftretenden Hautkrankheiten können vorbestehende wie die Schup- penflechte und die verschiedenen Ekzemformen während der Schwangerschaft Probleme bereiten. Nach unserem aktuellen Verständnis ist dabei die Th2-Situation der Schwangerschaft verantwortlich, dass sich die Schuppenflechte verbessert und ein atopisches Ekzem eher verschlechtert. Solide Untersuchungen zur Häufigkeit sowie dem klinischen Bild dieser Erkrankungen fehlen bis heute. Frau Dr. Renner gibt in ihrem Beitrag eine Übersicht der wichtigsten entzündlichen Erkrankungen und ihrer Bedeutung für Mutter und Kind.

\section{$\checkmark$ Isoliert in der Schwangerschaft auftretende Dermatosen bereiten manchmal differenzialdiagnostische Schwierigkeiten}

Auch Allergien können in der Schwangerschaft erstmalig auftreten oder exazerbieren, sodass sie die Lebensqualität der betroffenen Schwangeren deutlich beeinträchtigen. Diagnostische Untersuchungen sowie eine effiziente Therapie sind bei Schwangeren vor dem Hintergrund einer möglichen Fruchtschädigung kritisch zu sehen. Frau Priv.-Doz. Dr. Treudler schildert in ihrem Beitrag das Spektrum der allergischen Krankheiten sowie ihre Beeinflussbarkeit in dieser sehr kritischen und verletzlichen Lebensphase.

Infektionen der Haut gehören zu den häufigsten Erkrankungen der Schwangerschaft und sind in der Regel lokalisiert und damit topisch zu therapieren. Schwere Infektionen mit Allgemeinsymptomen bedürfen hingegen einer systemischen
Therapie, die sehr sorgfältig abgewogen und verfolgt werden sollte. Herr Prof. Müllegger aus Wien stellt hier anhand von exemplarischen viralen, bakteriellen und parasitären Erkrankungen die verschiedenen Indikationen und Behandlungsoptionen in der Schwangerschaft dar.

Die breite Palette von gutartigen Tumoren der Haut kann durch scheinbare Größenzunahme im Rahmen der Vermehrung des Bauchumfanges oder einer tatsächlichen Zunahme die Betroffenen stark beunruhigen. Je nach Ausmaß des Krankheitsbildes muss ggf. auch schon während der Schwangerschaft eine entsprechende Therapie eingeleitet werden. Dramatisch, aber glücklicherweise selten ist das Auftreten eines Melanoms in der Schwangerschaft, das angesichts der Prognose dringend therapiert werden sollte. Frau Dr. Erfurt-Berge gibt hier wichtige Informationen, um die Schwangere und ihre Familie in dieser extremen Situation adäquat zu versorgen und zu beraten.

Angesichts möglicher schädlicher Einflüsse ist die topische, aber insbesondere systemische Therapie bei Schwangeren stark eingeschränkt und kritisch abzuwägen. Auch aus forensischen Gründen sollte daher eine Systemtherapie nur in individuellen und mit den Betroffenen geklärten Fällen eingeleitet werden. Herr Prof. Wohlrab aus Halle zeigt die verschiedenen Möglichkeiten einer Topika- und Systemtherapie auf und diskutiert diese kritisch.

Dieses Leitthemenheft reflektiert damit die breite Palette von Hauterkrankungen in der Schwangerschaft und die Problematik des adäquaten Umganges mit ihnen. Sowohl Dermatologen, Allgemeinmedizinern und Geburtshelfern sollten 
diese Erkrankungen bekannt sein, um ggf. interdisziplinär frühzeitig eine adäquate Diagnostik und Therapie zum Wohle von Mutter und Kind einzuleiten.

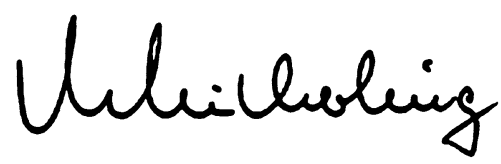

Prof. Dr. Michael Sticherling

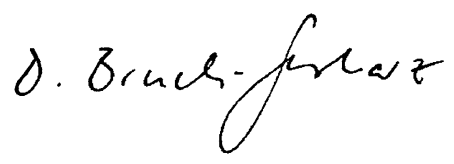

Prof. Dr. Daniela Bruch-Gerharz

\section{Korrespondenzadressen}

\section{Prof. Dr. M. Sticherling}

Hautklinik, Universitäts-
klinikum Erlangen
Hartmannstr. 14,
$\begin{aligned} & 91052 \text { Erlangen } \\ & \text { michael.sticherling@ } \\ & \text { uk-erlangen.de }\end{aligned}$

\section{Prof. Dr. D. Bruch-Gerharz}

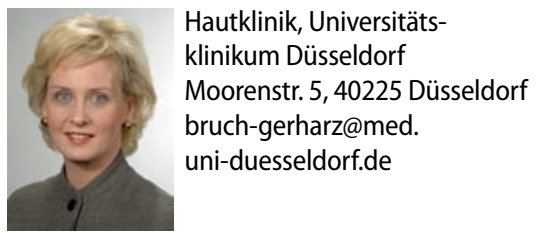

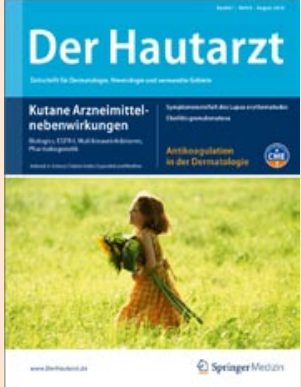

\section{Kasuistiken verfassen}

\section{Eindrucksvoll bebilderte Fallbeispiele} kompakt und strukturiert dargestellt

\author{
Sehr geehrte Autorin, \\ sehr geehrter Autor,
}

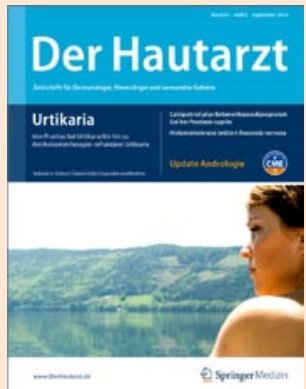

wir freuen uns, dass Sie die Zeitschrift "Der Hautarzt" mitgestalten möchten. Frei zur Publikation eingereichte Kasuistiken zeigen interessante Fallbeispiele und ungewöhnliche Krankheits- und Behandlungsverläufe. Damit unsere Leser den größtmöglichen Nutzen aus der Lektüre Ihres Beitrags ziehen können und umsetzbare Hinweise zu Diagnostik und Behandlung erhalten, möchten wie Ihnen mit der folgenden Checkliste gerne bei der Manuskripterstellung behilflich sein.

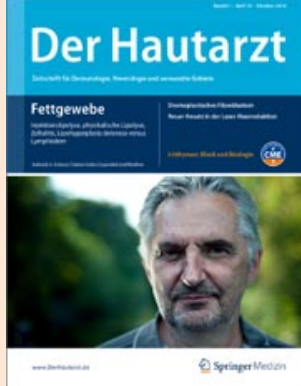

$\square$ Text bitte immer als Datei schicken (.doc oder .rtf)

$\square$ Komplette Anschrift des Korrespondenzautors mit Tel.Nr., Fax, E-Mail sowie Portraitfoto

$\square$ Gesamtumfang: max. 10.000 Zeichen inkl. Leerzeichen (Literatur, Tabellen und Abbildungslegenden bitte mitzählen)

$\square$ Kurzer, prägnanter Beitragstitel (ca. 50 Zeichen), ggf. erläuternder Untertitel

$\square$ Deutsche Zusammenfassung (max. 600 Zeichen inkl. Leerzeichen), 5 Schlüsselwörter

\section{Der Hautarzt}

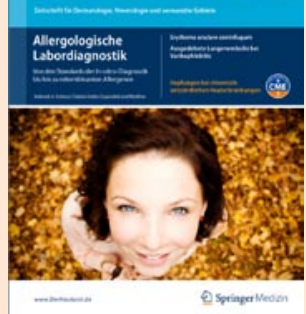

$\square$ Englischer Titel, englisches Abstract (max. 600 Zeichen inkl. Leerzeichen), 5 Keywords

$\square$ Gliederung in Anamnese, Befunde, Diagnose, Verlauf und Therapie, Diskussion

$\square$ Prägnante und möglichst kurze Zwischenüberschriften (max. 50 Zeichen).

$\square$ Fazit für die Praxis (max. 500 Zeichen)

$\square$ 3-4 Abbildungen pro Beitrag sind erwünscht

$\square$ möglichst kurze Abbildungslegenden

$\square$ Abbildungen und Tabellen im Text chronologisch erwähnen

$\square$ max. 10 Literaturzitate

Bitte senden Sie Ihren fertigen Beitrag an:

\section{Professor Dr. Alexander Kapp}

Klinik für Dermatologie, Allergologie und Venerologie, Medizinische Hochschule Hannover, Ricklinger Straße 5, 30449 Hannover,

E-Mail: freimooser.martina@mh-hannover.de

Weitere Informationen finden Sie unter

www.DerHautarzt.de 